\title{
Biomarcadores no Líquido Cefalorraquidiano no Desenvolvimento da Doença de Alzheimer: Uma Revisão Sistemática
}

\author{
Biomarkers in Cerebrospinal Fluid in Development Alzheimer's Disease: A Systematic \\ Review
}

\section{Biomarcadores en el Líquido Cefalorraquídeo en el Desarrollo de la Enfermedad de Alzheimer: Una Revisión Sistemática}

\author{
Ariane Billmann \\ Centro Universitário Ritter dos Reis \\ Marina Ferri Pezzini \\ Universidade Federal do Rio Grande do Sul
}

Julia Poeta ${ }^{1}$

Centro Universitário Ritter dos Reis

\begin{abstract}
Resumo
Objetivou-se identificar os artigos que analisam os biomarcadores tau total (T-tau), tau fosforilada (P-tau) e beta-amiloide ( $A B$ ) no líquido cefalorraquidiano (LCR) para o desenvolvimento da doença de Alzheimer (DA). Foi realizada uma revisão sistemática aplicando-se uma lógica de pesquisa nas bases de dados PubMed, SciELO e Medline, selecionando artigos conforme os critérios de inclusão e exclusão. Foram avaliados oito estudos clínicos, os quais demonstraram níveis de T-tau e P-tau elevados e níveis diminuídos de $A \beta$, entretanto nem todos encontraram significância estatística nesses achados. As dosagens dos biomarcadores $A \beta$, T-tau e P-tau em conjunto apresentam grande potencial diagnóstico no desenvolvimento da DA, contudo mais estudos são necessários para estabelecer valores de corte dos biomarcadores na evolução da doença.

Palavras-chave: Alzheimer, biomarcadores, líquido cefalorraquidiano, tau, amiloide
\end{abstract}

\begin{abstract}
This study aimed to identify studies that analyzed the total tau (T-tau), phosphorylated tau (P-tau) and beta-amyloid (A $\beta$ ) biomarkers in the cerebrospinal fluid (CSF) to the development of Alzheimer's disease (AD). The study is characterized as a systematic review, that was applied a search logic in PubMed, SciELO e Medline, selecting studies according to inclusion and exclusion criteria. Eight clinical studies were evaluated, and all of these found elevated levels of T-tau and P-tau and decreased levels of $A \beta$, but not all studies found statistical significance in these findings. The dosage of the biomarkers $A \beta, T$-tau, and P-tau together present a great diagnostic potential of AD; however, more studies are needed to establish cutoff values of these biomarkers in disease progression.
\end{abstract}

Keywords: Alzheimer's, biomarkers, cerebrospinal fluid, tau, amyloid

\section{Resumen}

Se objetivó identificar los artículos que analizaran los biomarcadores tau total (T-tau), tau fosforilada (P-tau) y beta-amiloide (A $\beta$ ) en el líquido cefalorraquídeo (LCR) para el desarrollo de la enfermedad de Alzheimer (DA). Se realizó una revisión sistemática aplicando una lógica de investigación en las bases de datos PubMed, SciELO y Medline, seleccionando artículos conforme a los criterios de inclusión y exclusión. Se evaluaron ocho estudios clínicos, los cuales se encontraron niveles de T-tau y P-tau elevados y niveles disminuidos de $A \beta$, pero no todos encontraron significación estadística en estos hallazgos. Las dosificaciones de los biomarcadores $A \beta$, T-tau y P-tau en conjunto presentan un gran potencial diagnóstico en el desarrollo de la DA, pero más estudios son necesarios para establecer valores de corte de los biomarcadores en la evolución de la enfermedad.

Palabras clave: Alzheimer, biomarcadores, líquido cefalorraquídeo, tau, amiloide

\footnotetext{
${ }^{1}$ Endereço de contato: Rua Orfanotrófio, 555, Santa Tereza, Porto Alegre, RS, CEP 90840-440. E-mail: julia_poeta@uniritter.edu.br
} 
As doenças demenciais representam um crescente desafio para as áreas médicas, econômicas e sociais, e a doença de Alzheimer (DA) é a causa mais comum de demência associada ao avanço da idade. A Organização Mundial de Saúde (OMS) define DA como uma doença neurodegenerativa, severa, progressiva, de etiologia desconhecida e que causa declínio dos processos cognitivos de memória, afetando a qualidade de vida do paciente e de seus familiares (World Health Organization, 2018). Além da perda de memória, à medida que a doença progride, ocorre acometimento da mobilidade, linguagem e reconhecimento, podendo levar a casos de depressão e mudanças de personalidade (Corrêa, Starling, Teixeira, Caramelli, \& Silva, 2011; Marques, \& Lapa, 2018; Pedroso et al., 2018). Mundialmente, cerca de 50 milhões de pessoas sofrem de demência e 10 milhões de novos casos por ano são diagnosticados (Organizacón Mundial de la Salud, 2019). No Brasil, estima-se que há cerca de 1,2 milhão de casos, a maior parte deles ainda sem diagnóstico (Associação Brasileira de Alzheimer, s.d.).

O diagnóstico da DA é, normalmente, feito com base em exames clínicos, laboratoriais e de neuroimagem cerebral, na avaliação do histórico familiar e nos testes neuropsicológicos, seguindo as normas do National Institute of Neurological and Communicative Disorders and Stroke (NINCDS) e da Alzheimer's Disease and Related Disorders Association (ADRDA) (Associação Brasileira de Alzheimer, s.d.; Frota et al., 2011; Nitzsche, Moraes, \& Tavares, 2015). Não se trata de um diagnóstico específico, e sim um meio de excluir outras possíveis causas para a demência, como acidente vascular cerebral (AVC), comprometimento cerebral leve (CCL), tumores ou efeitos colaterais medicamentosos. Dessa maneira, de acordo com as causas excluídas para demência, a DA pode ser classificada em: a) DA possível: com sintomas não característicos; b) DA provável: com sintomas característicos; c) DA definitiva: com análise de tecido cerebral após o óbito, sendo o diagnóstico conclusivo a análise microscópica de tecido cerebral. Diante dessas dificuldades, estudos têm sido realizados com o objetivo de identificar diferentes métodos, incluindo a dosagem de proteínas para auxiliar o diagnóstico e, possivelmente, prever a doença com antecedência. As principais proteínas, conhecidas como biomarcadores, são: a beta-amiloide ( $A \beta-42)$, a tau total (T-tau) e tau fosforilada (P-tau). Elas representam um tipo de "assinatura patológica" da DA e são encontradas no líquido cefalorraquidiano (LCR), dando, assim, a possibilidade de um diagnóstico preciso e diferencial. No entanto sua utilização no diagnóstico pré-clínico foi recomendada somente em pesquisas até o momento, pelo fato da ausência de dados confirmatórios, do acesso restrito à coleta do LCR e dosagens laboratoriais com diferentes pontos de corte (Frota et al., 2011; McKhann et al., 2011; Nitzsche et al., 2015; Sereniki, \& Vital, 2008).

$A A \beta$ é um fragmento precursor da proteína precursora de amiloide (APP), não tem função conhecida e é a principal constituinte das placas senis. Na DA, há uma superprodução desse fragmento e formação de moléculas acumuladas como oligômeros, alterando a comunicação com os neurônios. Como sua eliminação é por meio do LCR, a deposição no parênquima cerebral faz com que sua concentração diminua (Blennow et al., 2015; Dubois et al., 2014; Lashley et al., 2018). Já a proteína tau encontra-se dentro dos neurônios e é responsável pela estabilização dos microtúbulos no sistema de transporte neurítico. Na DA, ocorre a hiperfosforilação e agregação da tau, formando emaranhados neurofibrilares 
intracelulares (ENF) que desencadeiam morte neuronal. Com a degeneração dos neurônios, a tau é eliminada pelo LCR, aumentando a concentração de P-tau nele (Blennow et al., 2015; Dubois et al., 2014). A utilização da pesquisa da A $\beta$ no LCR para o diagnóstico de DA tem se apresentado bastante sensível e específica. Ainda, quando analisada conjuntamente com proteína tau, a sensibilidade tende a aumentar, permitindo a diferenciação entre DA e outros tipos de demências, como a demência frontotemporal (DFT) (Schoonenboom et al., 2004).

Como não há cura para a DA, foram desenvolvidos tratamentos paliativos a fim de evitar que a doença progrida. O diagnóstico precoce resulta num tratamento que retarda a progressão da doença e, consequentemente, traz melhoria da qualidade de vida. Dessa maneira, a utilização dos biomarcadores visa a um diagnóstico na fase pré-clínica, abrindo possibilidade para tratar/retardar um futuro dano neurológico. Após um possível diagnóstico, há basicamente dois tipos de tratamentos: os farmacológicos - que atuam na inibição de colinesterases e elevam a disponibilidade de neurotransmissores como acetilcolina (ACh) na fenda sináptica, evitando a toxicidade do glutamato para o neurônio; e os não farmacológicos - definidos por atividades que estimulam o cérebro, como musicoterapia, arteterapia, psicoterapia e outras atividades estimulantes. No Brasil, os fármacos que estão aprovados pela Agência Nacional de Vigilância Sanitária (ANVISA) são: Tacrina, Rivastigmina, Donepezila, Memantina e Galantamina. A administração e a dosagem dos fármacos variam conforme o estágio da doença (moderado a grave), podendo iniciar em $5 \mathrm{mg} /$ dia (doença leve a moderadamente grave) a $10 \mathrm{mg} /$ dia (doença moderadamente grave a grave) (Engelhardt et al., 2005; Forlenza, 2005).

Diante dos fatos apresentados, esta revisão bibliográfica tem como objetivo buscar os principais estudos publicados na literatura que avaliaram a utilização dos biomarcadores no LCR (Aß, P-tau e T-tau) para o diagnóstico da doença de Alzheimer, visto que essas são as principais proteínas marcadoras de lesão neurológica, possibilitando, assim, um diagnóstico precoce de uma patologia considerada de diagnóstico difícil, tardio e, muitas vezes, inconclusivo.

\section{Método}

O presente estudo foi realizado por meio de uma revisão sistemática da literatura de ensaios clínicos sobre o uso de biomarcadores no líquido cefalorraquidiano para o diagnóstico de Alzheimer. O critério de inclusão adotado foi de publicações cujas temáticas abordassem a utilização e dosagem de marcadores de LCR, T-tau, P-tau e A $\beta$ no diagnóstico de pacientes com suspeita da doença de Alzheimer, entre os anos de 2011 e 2018. Além disso, deveriam descrever como desfecho principal as variações desses biomarcadores na DA. Artigos sem essas informações foram excluídos, assim como artigos de revisão ou que não estavam disponíveis na íntegra para a leitura. Primeiramente, as publicações encontradas nas buscas passaram por análise de títulos e resumos, sendo esses selecionados e lidos de maneira detalhada. Foram excluídas da seleção publicações cujo objetivo era a dosagem dos biomarcadores para avaliações farmacológicas em andamento. A análise seguiu referencial teórico, em que foi inclusa leitura exploratória, interpretativa e seletiva do material coletado. 
A coleta de dados foi realizada durante os meses de março e maio de 2018, nas bases de dados eletrônicos SciELO, Medline e PubMed, nos idiomas português, inglês e espanhol, contemplando artigos entre os anos de 2010 e 2018. Para a busca, utilizaram-se quatro descritores e as combinações deles em inglês, sendo eles: Alzheimer, biomarcadores, tau, amiloide, cerebroespinhal (Alzheimer, biomarkers, tau, amyloid, cerebrospinal). A fim de facilitar a triagem dos estudos, utilizou-se o software específico para elaboração de revisões sistemáticas, o State of the Art through Systematic Review (StArt). Mediante relação dos artigos pré-selecionados e importados para o StArt, três pesquisadores, de forma independente, fizeram o rastreamento dos estudos duplicados, excluídos e potencialmente elegíveis à revisão, por meio da leitura dos títulos e resumos.

Como critérios de elegibilidade dos artigos, foram adotadas três etapas para inclusão e exclusão, sendo: a) artigos selecionados igualmente pelos três pesquisadores foram incluídos automaticamente; b) artigos não selecionados ou selecionados por apenas um pesquisador foram excluídos automaticamente; c) artigos incluídos por dois pesquisadores foram analisados por um revisor e, no caso de enquadramento, foram incluídos.

No total, foram recuperados 2.772 artigos (PubMed: 1.448; Medline: 1.319; SciELO: 5, por meio das buscas eletrônicas. Inicialmente, 121 artigos foram excluídos em virtude de duplicidade e 2.515 por não se enquadrarem nos critérios de inclusão, mediante leitura do título e resumo. Assim, foram considerados elegíveis 136 estudos para análise integral do artigo. Desses, 128 foram excluídos por não se enquadrarem nos desfechos propostos, sendo selecionados oito estudos (Figura 1). 


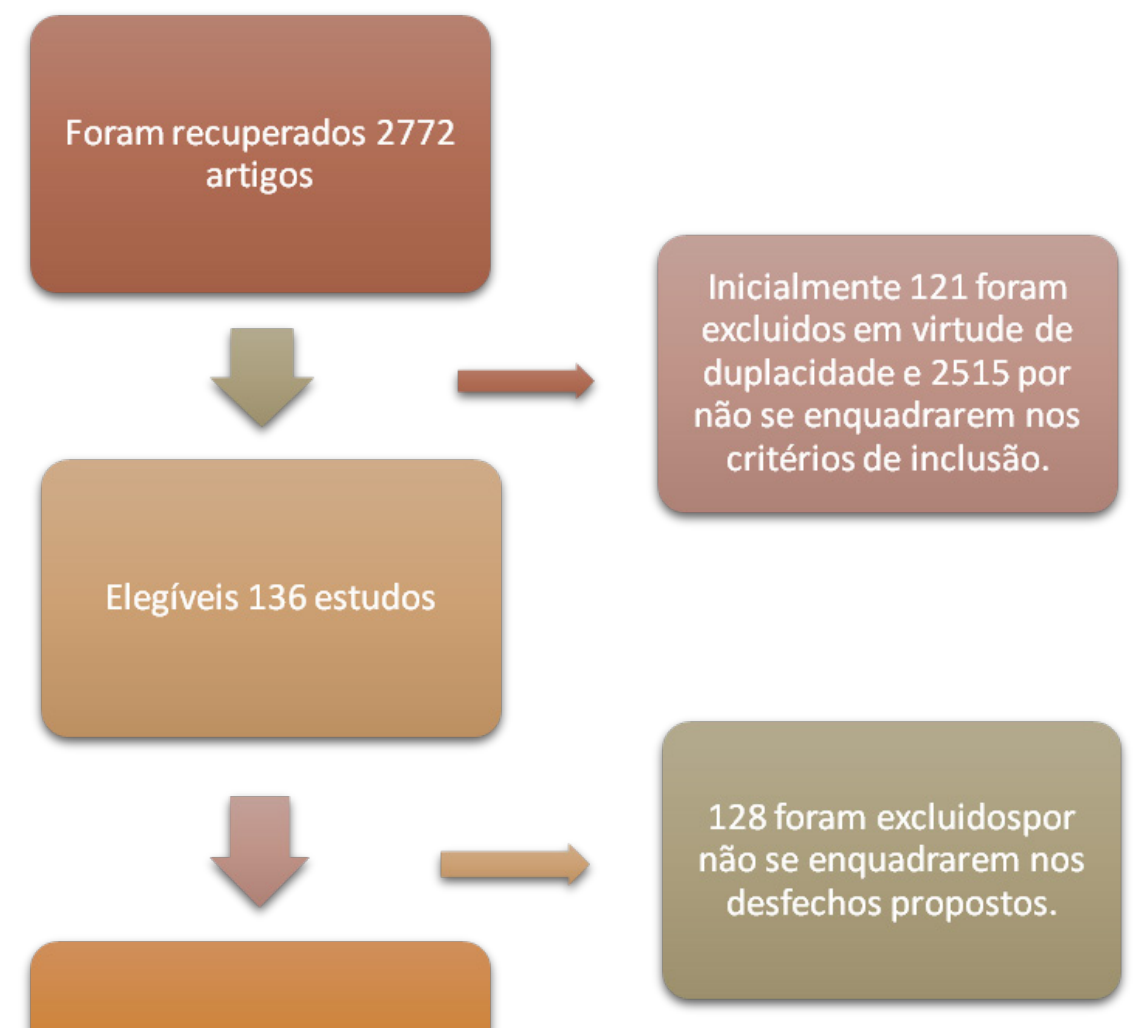

Selecionados 8 estudos

Figura 1. Desenho do estudo e seleção de artigos.

Fonte: Próprias autoras.

\section{Resultados e Discussão}

Nos estudos selecionados (Tabela 1), foram realizadas dosagens dos biomarcadores (T-tau, P-tau e A $\beta$ ) em LCR de pacientes com DA possível, DA provável, com alguma anormalidade cognitiva e indivíduos controle, que não apresentavam alterações cognitivas nem DA. Estudos como os de Trombetta et al. (2018), Magalhães et al. (2015), Corrêa et al. (2011) e Radanovic, Stella, Silva, Talib e Forlenza (2017) observaram valores elevados de proteína tau e níveis reduzidos de proteína $A \beta$ no LCR de pacientes com DA, porém esses resultados não foram estatisticamente significativos. Trombetta et al. (2018) relataram que cada um dos biomarcadores testados (T-tau, $A \beta-38, A \beta-40$ e $A \beta-42$ ) demonstrou quantificação consistente, confiável e estável, estabelecendo potencial para uso em estudos exploratórios. Esses dados estão de acordo com o estudo de Pan et al. (2015), o qual também demonstrou desempenho analítico consistente e estável, no entanto nenhum deles foi considerado específico para a DA, e sim marcadores gerais de neurodegeneração. Dessa maneira, esses dados podem ser úteis como auxílio complementar ou, até mesmo, para quantificar o grau de degeneração ativa no momento da coleta. 


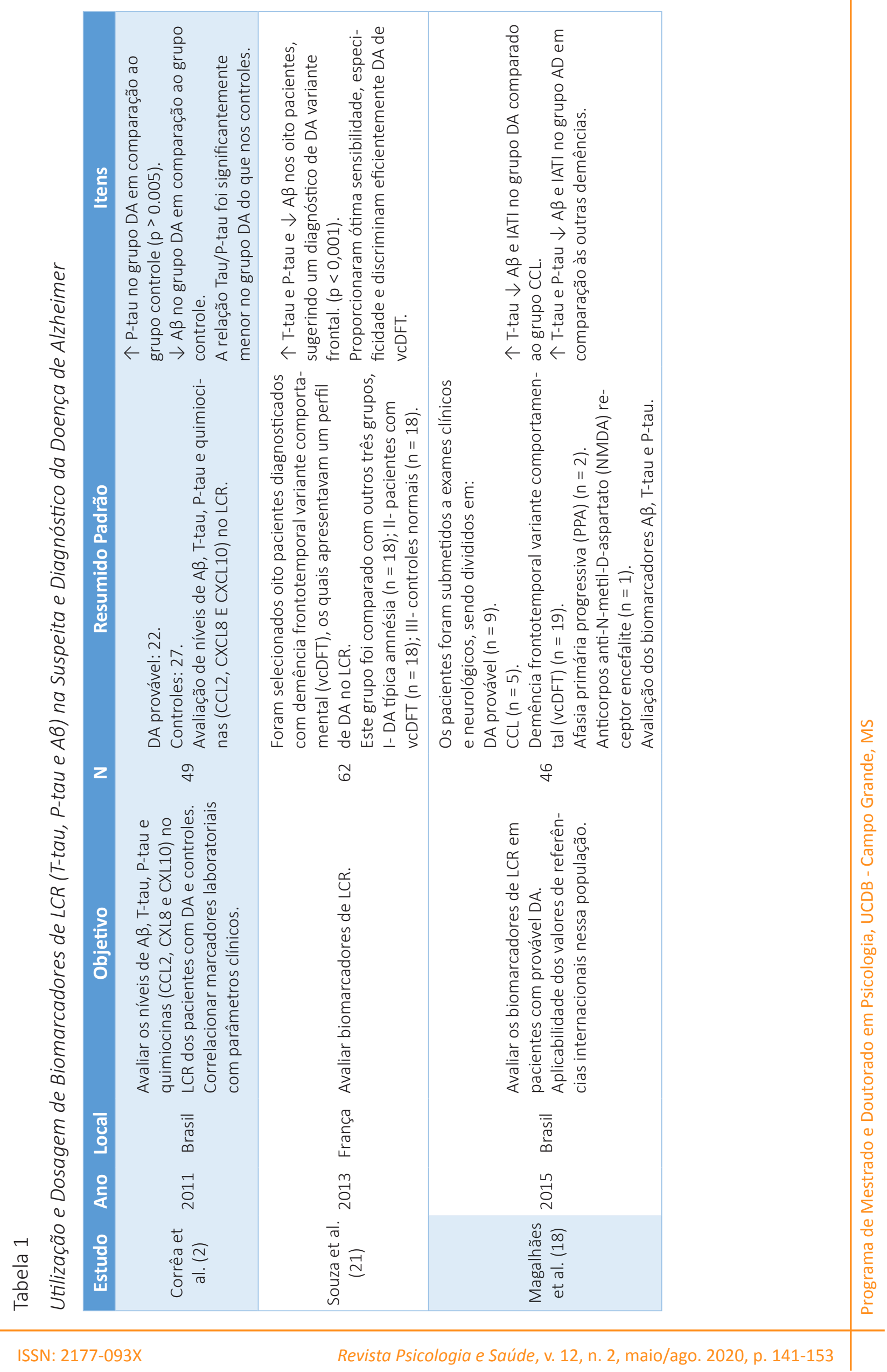




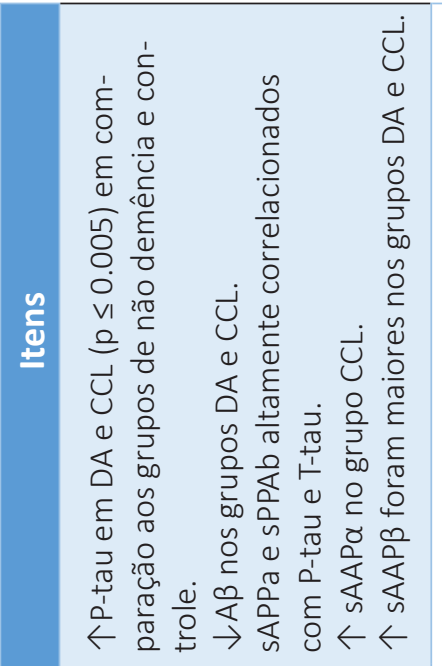

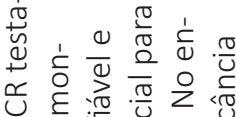

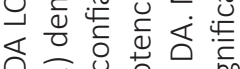

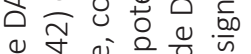

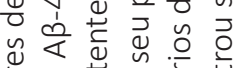

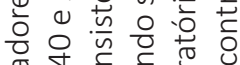

先

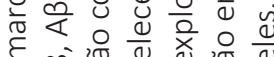

है

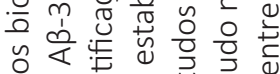

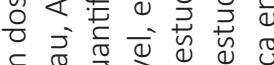

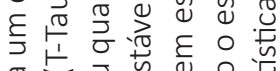

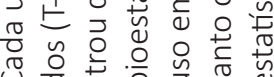

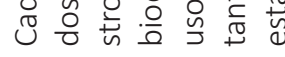

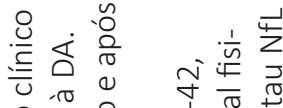

0

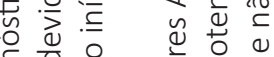

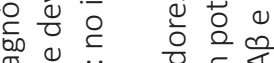

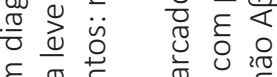

ह $\frac{\pi}{\mathrm{C}}$

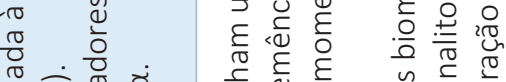

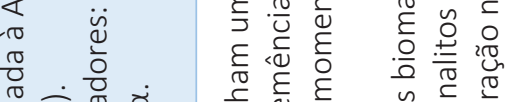

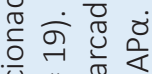

प 11 更

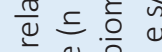

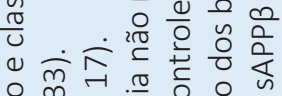
iक्य 11 "I

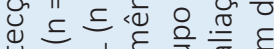

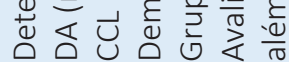

$z$

๙ั

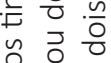

응 을

$\frac{n \pi}{\pi} \overline{0} \frac{\pi}{\sigma}$

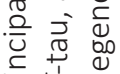

言

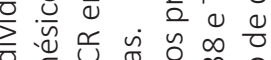

$\subseteq$ ह

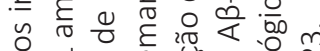

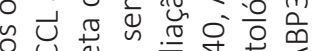

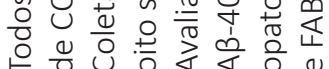

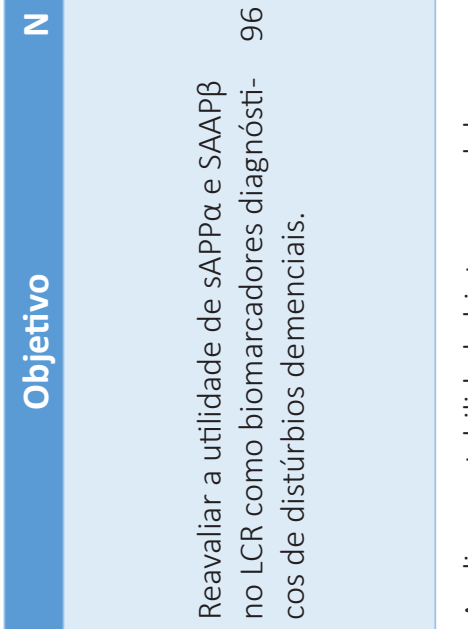

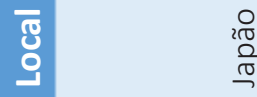

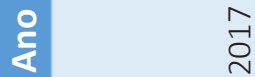

ㅇ

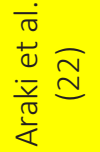

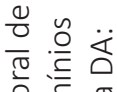

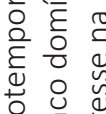

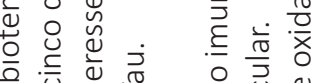

ब U

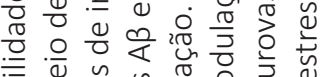

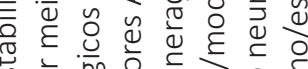

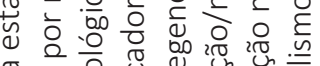

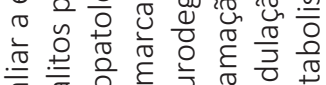

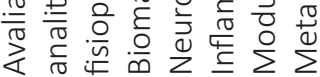

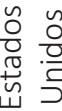

离

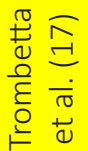

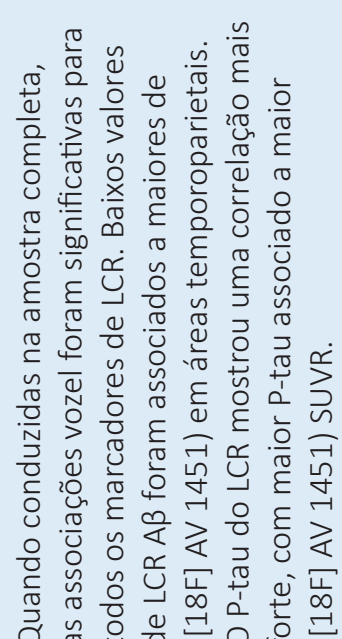

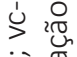

它

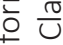

ơ

宁

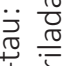

ㄴ.

元 恼

告

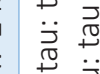

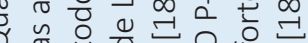

这岌

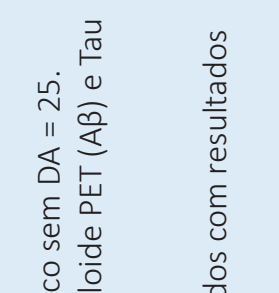

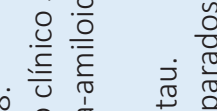

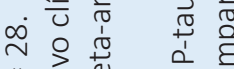

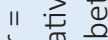

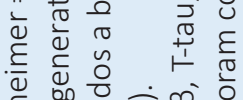

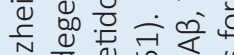

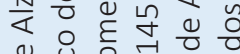

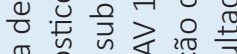

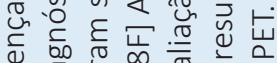

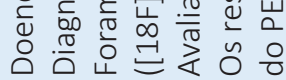

กำ

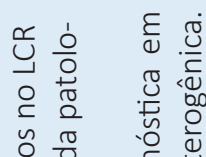

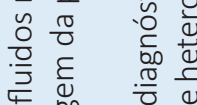

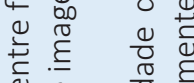

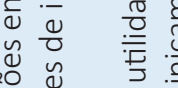

20

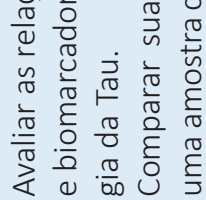

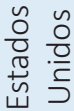

官

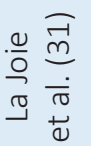

हิ

范 $\frac{\pi}{2}$

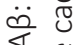

ㅎํㅇ

들

단 $\frac{0}{\sqrt{0}}$

흐은

$\frac{\sqrt[\pi]{0}}{0} \frac{0}{2}$

융 돈

믈

붠

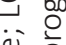

बें

:

范

윤

造这

है

을 है

ठิ

نे है

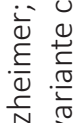

$\frac{\sqrt{2}}{\frac{\pi}{2}}$

$\frac{0}{8}$

들 है

वे 웅

疋

일

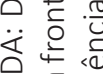

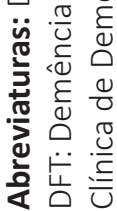


Magalhães et al. (2015) e Radanovic et al. (2017) avaliaram os marcadores em pacientes com DA, com CCL e com outras demências comparando com grupos controle. Eles encontraram valores elevados para T-tau e P-tau, e reduzidos para A $\beta$ no grupo DA, quando comparado aos grupos CCL e de outras demências. No entanto os estudos ressaltam que nenhum biomarcador forneceu sensibilidade e especificidade suficientes para o diagnóstico de DA quando avaliado isoladamente, o que justifica a sua solicitação e análise em conjunto. Além disso, eles sugerem uma complementação do diagnóstico final, sempre que possível, por meio de exames de imagem, como a ressonância magnética (RM) e a tomografia computadorizada por emissão de pósitrons (PET). Os achados de Correa et al. (2011) são consistentes com os outros estudos anteriores (Magalhães et al., 2015; Radanovic et al., 2017), pois mostram níveis aumentados de P-tau e níveis diminuídos de $A \beta$ no LCR de pacientes com DA.

Entretanto outros estudos encontraram relevância significativa entre as variações no LCR dos biomarcadores de indivíduos com e sem a DA (Araki et al., 2017; De Souza et al., 2013). De Souza et al. (2013) avaliaram os biomarcadores no LCR em pacientes com demência frontotemporal variante comportamental (vcDFT) e obtiveram significativas diferenças entre os níveis de $A \beta$, T-tau e P-tau, para o diagnóstico de DA. Outros dados obtidos por meio de diferentes tipos de materiais para análise, como biológico, genético ou de confirmação patológica, também relataram apresentações atípicas focais da DA imitando vcFTD (Yew, Alladi, Shailaja, Hodges, \& Hornberger, 2013). Um estudo de Piguet, Hornberger, Mioshi e Hodges (2011) defende que demência frontotemporal (DFT) é a segunda mais frequente causa de demência degenerativa em pacientes com mais de 65 anos, e é pouco frequente identificar casos de DA em pacientes com esse tipo de demência.

La Joie et al. (2018) utilizaram o radiofármaco (PET [18F] AV1451) que localiza filamentos de tau, que incluem emaranhados neurofibrilares, PET A e biomarcadores de LCR. Contrapondo o estudo de Gordon et al. (2016) que indicou relações fracassadas com tais biomarcadores, La Joie et al. (2018) encontraram relevância entre eles. Níveis reduzidos de $A \beta$ no LCR foram associados a maiores de radiofármaco utilizado em áreas temporoparietais. Dosagens de P-tau demostraram uma correlação mais forte, com maior relação da P-tau associada a maior ([18F] AV 1451) SUVR. Os presentes dados defendem que a incorporação de P-tau e [F18] AV1451- PET no LCR são biomarcadores confiáveis para a detecção de P-tau numa amostra clínica.

Araki et al. (2017) avaliaram os níveis A $\beta$, T-tau e P-tau e ainda incluíram as secretases SAAP $\alpha$ SAAP $\beta$, com a possibilidade de serem biomarcadores específicos da DA. Como marcadores diagnósticos estabelecidos de DA e CCL, os níveis de P-tau foram significativamente elevados nos grupos DA e CCL em comparação com os grupos de não demência e controle da doença; e os níveis de A $\beta$ foram reduzidos nos grupos DA e CCL. Ambos sAPP $\alpha$ e sAPP $\beta$ foram altamente correlacionados com P-tau e T-total, sugerindo que ambos os SAPPs refletem alterações neuropatológicas no cérebro. Os níveis de SAAP $\alpha$ foram especificamente mais elevados no grupo CCL em comparação com os grupos de não DA e controle, e os níveis de SAAP $\beta$ foram maiores nos grupos DA e CCL em comparação com os outros grupos. Como ambos os SAPPs têm bom poder discriminativo para o diagnóstico de $\mathrm{CCL}$, este estudo defende que os SAAPs, no LCR, são biomarcadores potencialmente úteis e complementares para o diagnóstico precoce e preciso de distúrbios demenciais. 
Albert et al. (2018) avaliaram a estabilidade temporal dos biomarcadores LCR A $\beta$, P-tau, volume hipocampal e entorrinal do córtex, resultados dos testes cognitivo e genótipo APOE em pacientes normais, com três durações de acompanhamento (5, 7 e 10 anos) após linha de base. Cada uma das variáveis individuais foi significativamente associada com a progressão da cognição normal para o início dos sintomas de $C C L$, exceto para a $A \beta$ no $L C R$, que não foi significativo. Esses resultados podem facilitar o planejamento de estudos de intervenções voltados para a base pré-analítica da doença de Alzheimer.

Stouta et al. (2017) relacionaram os biomarcadores de DA com o tempo em que idosos deixariam de dirigir. As análises de A $\beta$, T-tau e P-tau foram realizadas no LCR; os idosos com elevação de T-tau e P-tau, bem como diminuição de $A \beta$, pararam de dirigir, aproximadamente, duas vezes mais rápido que a taxa por ano dos indivíduos que apresentaram níveis normais dos biomarcadores analisados. Além disso, foi calculada a relação T-tau/ $A \beta$ e P-tau/A $\beta$, as quais estavam elevadas, indicando DA pré-clínica. Já no estudo de Choi, Lohman e Mezuk (2014) em que avaliaram motoristas idosos durante dez anos em relação à mudança no funcionamento cognitivo e ao tempo de cessação, idosos sem mobilidade motora apresentaram pior funcionamento cognitivo no início do estudo e, durante o acompanhamento, apresentaram declínio cognitivo acelerado em relação aos condutores ativos. No entanto a avaliação desse foi realizada por autorrelato e não realizou a dosagem dos biomarcadores.

Diante dos resultados obtidos, há dificuldade em definir os valores e a relação dos biomarcadores pesquisados com a DA, visto que a maioria dos biomarcadores analisados se apresentaram alterados na DA e em outras demências quando comparados com os controles, no entanto não se apresentaram capazes de distinguir DA de outras demências.

\section{Considerações Finais}

Tendo em vista os resultados observados, os biomarcadores de LCR (Aß, T-tau e P-tau) apresentam, desta forma, um grande potencial diagnóstico para a DA, já que são considerados "assinaturas patológicas" de neurodegeneração e podem ser mensurados no LCR. Embora esses biomarcadores sejam altamente úteis, eles ainda apresentam limitações, como a variabilidade intralaboratorial, substancial sobreposição com outras formas de demência, invasividade do procedimento e alto custo. Novos estudos ajudarão a definir os estágios fisiopatológicos da DA e identificar os indivíduos com risco aumentado de declínio cognitivo. Um diagnóstico preciso e precoce da DA é essencial para auxiliar a equipe médica e selecionar o atendimento ideal ao paciente, sendo fundamental nos atuais ensaios clínicos.

Nosso estudo apresenta algumas limitações, sendo uma delas o reduzido número de estudos analisados, que nos trazem conclusões questionáveis quanto ao uso dos biomarcadores na prática clínica. Além disso, nossa pesquisa trata-se de uma revisão sistemática, a qual utilizou apenas métodos sistemáticos, e não estatísticos, para identificar, selecionar e avaliar criticamente os estudos. Os resultados dos estudos apresentaram significância clínica, entretanto nem todos apresentaram significância estatística. 


\section{Referências}

Albert, M., Zhu, Y., Moghekar, A., Mori, S., Miller, M., Soldan, A., . . W Wang M. (2018). Predicting progression from normal cognition to mild cognitive impairment for individuals at 5 years. Brain Journal, 141(3), 877-887. Disponível em https://academic.oup.com/ brain/article/141/3/877/4818093

Araki, W., Hattori, K., Kanemaru, K., Yokoi, Y., Omachi, Y., Takano, H., ... Mizusawa, H. (2017). Re-evaluation of soluble APP- $\alpha$ and APP- $\beta$ in cerebrospinal fluid as potential biomarkers for early diagnosis of dementia disorders. Biomarker research, 5(28). Disponível em https:// www.ncbi.nlm.nih.gov/pmc/articles/PMC5610422/pdf/40364_2017_Article_108.pdf

Associação Brasileira de Alzheimer. (s.d.). O que é Alzheimer? Disponível em http://abraz.org. br/web/sobre-alzheimer/o-que-e-alzheimer/

Blennow, K., Dubois, B., Fagan, A. M., Lewczuk, P., Leon, M. J, \& Hampel, H. (2015). Clinical utility of cerebrospinal fluid biomarkers in the diagnosis of early Alzheimer's disease. Alzheimer's e Dementia, 11(1), 58-69. Disponível em https://www.alzheimersanddementia.com/ article/S1552-5260(14)00066-1/fulltext

Choi, M., Lohman, M. C., \& Mezuk, B. (2014) Trajectories of cognitive decline by driving mobility: Evidence from the Health and Retirement Study. Int J Geriatr Psychiatry, 29(5), 447-53. Disponível em https://onlinelibrary.wiley.com/doi/full/10.1002/gps.4024

Corrêa, J. D., Starling, D., Teixeira., A. L., Caramelli, P., \& Silva T. A. (2011). Chemokines in CSF of Alzheimer's disease patients. Arquivos de NeuroPsiquiatria, 69(3), 455-459. Disponível em http://www.scielo.br/scielo. php?script=sci_arttext\&pid=S0004282X2011000400009\&Ing=en\&nrm=iso\&tlng=en

De Souza, L. C., Bertoux, M., Funkiewiez, A., Samri, D., Azuar, C., Habert, ... Dubois, B. (2013). Frontal presentation of Alzheimer's disease: A series of patients with biological evidence by CSF biomarkers. Dementia \& Neuropsychologia, 7(1), 66-74. Disponível em: https:// www.ncbi.nlm.nih.gov/pmc/articles/PMC5619547/

Dubois, B., Feldman, H. H., Jacova, C., Hampel, H., Molinuevo, J. L., Blennow, K., ... Cummings, J. L. (2014). Advancing research diagnostic criteria for Alzheimer's disease: The IWG-2 criteria. The Lancet Neurology, 13(6), 614-29. Disponivel em https://www.thelancet.com/ journals/laneur/article/PIIS1474-4422(14)70090-0/fulltext\#\%20

Engelhardt, E., Brucki, S. M., Cavalcanti, J. L. S., Forlenza, O. V., Laks, J., \& Vale, F. A. C (2005). Tratamento da doença de Alzheimer: recomendações e sugestões do Departamento Científico de Neurologia Cognitiva e do Envelhecimento da Academia Brasileira de Neurologia. Arquivos de Neuro-Psiquiatria, 63(4), 1104-1112. Disponível em http://www. scielo.br/scielo.php?script=sci_arttext\&pid=\$0004-282X2005000600035

Forlenza, O. V. (2005). Tratamento farmacológico da doença de Alzheimer. Archives of Clinical Psychiatry, 32(3), 137-148. Disponível em http://www.scielo.br/scielo. php?script=sci_arttext $\&$ pid $=$ S0101-60832005000300006

Frota, N. A. F., Nitrini, R., Damasceno, B. P., Forlenza, O., Tosta, E. D., Silva, A. B., ... Magaldi, R. M. (2011). Critérios para o diagnóstico de doença de Alzheimer. Dementia \& Neuropsychologia, 5(1), 5-10. Disponível em http://www.demneuropsy.com.br/imageBank/pdf/v5s1a02.pdf Gordon, B. A., Friedrichsen, K., Brier, M., Blazey, T., Su, Y., Christensen, J., . . Benzinger T. L. (2016). The relationship between cerebrospinal fluid markers of Alzheimer pathology 
and positron emission tomography tau imaging. Brain, 139(8), 2249-2260. Disponível em https://academic.oup.com/brain/article/139/8/2249/1753889

La Joie, R., Bejanin, A., Fagan, A., Ayakta, N., Baker, S., Bourakova, ... Rabinovici, G. D. (2018). Associations between [18F] AV1451 tau PET and CSF measures of tau pathology in a clinical sample. Neurology, 90(4), 282-290. Disponível em https://www.ncbi.nlm.nih.gov/ pmc/articles/PMC5798657/pdf/NEUROLOGY2017827394.pdf

Lashley, T., Schott, J., Weston, P., Murray, C. E., Wellington, H., Keshavan, A., . . . Zetterberg, H. (2018). Molecular biomarkers of Alzheimer's disease: Progress and prospects. Disease Models \& Mechanisms, 11(5), Disponível em https://www.ncbi.nlm.nih.gov/pmc/articles/ PMC5992610/

Magalhães, C., Figueiró, M., Fraga, V., Mateo, E., Toledo, A., Carvalho, M. G., . . . Gomes, K. B. (2015). Cerebrospinal fluid biomarkers for the differential diagnosis of Alzheimer's disease. Jornal Brasileiro de Patologia e Medicina Laboratorial, 51(6), 376-382. Disponível em http://www.scielo.br/pdf/jbpml/v51n6/1676-2444-jbpml-51-06-0376.pdf

Marques, A. F., \& Lapa, T. A. (2018). Anestesia e doença de Alzheimer. Revista Brasileira de Anestesiologia, 68(2), 174-182. Disponível em http://www.scielo.br/pdf/rba/v68n2/ pt_0034-7094-rba-68-02-0174.pdf

McKhann, G. M., Knopman, D. S., Chertkow, H., Hyman, B. T., Jack, C. R., Kawas, C. H., ... Phelps, C. H. (2011). The diagnosis of dementia due to Alzheimer's disease: Recommendations from the National Institute on Aging and Alzheimer's Association workgroup. Alzheimers Dement, 7(3), 263-9. Disponível em https://www.ncbi.nlm.nih.gov/pmc/articles/ PMC3312024/pdf/nihms363310.pdf

Nitzsche, B. A., Moraes, H. P., \& Tavares, A. R. (2015). Doença de Alzheimer: Novas diretrizes para o diagnóstico. Revista Médica de Minas Gerais, 25(2), 237-243. Disponível em https:// docplayer.com.br/4407071-Doenca-de-alzheimer-novas-diretrizes-para-o-diagnostico. html

Organizacón Mundial de la Salud. (2019). Demencia. Disponível em https://www.who.int/ es/news-room/fact-sheets/detail/dementia

Pan, C., Korff, A., Galasko, D., Ginghina, C., Peskind, E., Li, G., .. . Zhang, J. (2015). Diagnostic Values of Cerebrospinal Fluid T-Tau and $A \beta_{42}$ using Meso Scale Discovery Assays for Alzheimer's Disease. Journal of Alzheimer's Disease, 45(3), 709-19. Disponível em https:// www.ncbi.nlm.nih.gov/pmc/articles/PMC4517668/

Pedroso, R. V., Corazza, D. I., Andreatto, C., da Silva, T., Costa, J., \& Santos-Galduróz, R. F. (2018). Cognitive, functional and physical activity impairment in elderly with Alzheimer's disease. Dementia \& Neuropsychologia, 12(1), 28-34. Disponível em https://www.ncbi. nlm.nih.gov/pmc/articles/PMC5901246/

Piguet, O., Hornberger, M., Mioshi, E., \&Hodges,J. R. (2011). Behavioural-variantfrontotemporal dementia: Diagnosis, clinical staging, and management. The Lancet Neurology, 10(2), 162172. Disponível em https://www.ncbi.nlm.nih.gov/pubmed/21147039

Radanovic, M., Stella, F., Silva, L. G., Talib, L. L., \& Forlenza, O. V. (2017). Increased CSF levels of total Tau in patients with subcortical cerebrovascular pathology and cognitive impairment. Dementia \& Neuropsychologia, 11(4), 419-425. Disponível em: https://www. ncbi.nlm.nih.gov/pmc/articles/PMC5770001/ 
Schoonenboom, N. S., Pijnenburg, Y. A., Mulder, C., Rosso, S. M., Van Elk, E. J., Van Kamp, ... Scheltens, P. (2004). Amyloid beta (1-42) and phosphorylated tau in CSF as markers for early-onset Alzheimer disease. Neurology, 62(9), 1580-1584. Disponível em https://www. ncbi.nlm.nih.gov/pubmed/15136685

Sereniki, A., \& Vital, M. A. (2008). A doença de Alzheimer: Aspectos fisiopatológicos e farmacológicos. Revista de Psiquiatria do Rio Grande do Sul, 30(1). Disponível em http:// www.scielo.br/scielo.php?script=sci_arttext\&pid=\$0101-81082008000200002

Stouta, S., Babulala, G., Maa, C., Carrd, D., Heada, D., Granta, E., ... Roe, C. M. (2017). Driving cessation over a 24-year period: Dementia severity and cerebrospinal fluid biomarkers. Alzheimer's \& Dementia, 14(5), 610-616. Disponível em https://www.ncbi.nlm.nih.gov/ pubmed/29328928

Trombetta, B. A., Carlyle, B. C., Koenig, A. M., Shaw, L. M., Trojanowski, J. Q., Wolk, D. A., ... Arnold, S. E. (2018). The technical reliability and biotemporal stability of cerebrospinal fluid biomarkers for profiling multiple pathophysiologies in Alzheimer's disease. Plos One, 13(3). Disponível em https://www.ncbi.nlm.nih.gov/pmc/articles/PMC5837100/

World Health Organization. (2018). The ICD-10 classification of mental and behavioural disorders: clinical descriptions and diagnostic guidelines. Genebra: WHO. Disponível em https://www.who.int/classifications/icd/en/bluebook.pdf

Yew, B., Alladi, S., Shailaja, M., Hodges, J. R., \& Hornberger, M. (2013). Lost and forgotten? Orientation versus memory in Alzheimer's disease and frontotemporal dementia. Journal of Alzheimer's Disease, 33(2), 473-481.

Recebido em: 19/02/2019

última Revisão: 07/05/2019

Aceite Final: 03/07/2019

\section{Sobre as autoras:}

Ariane Billmann- Graduada em Biomedicina pelo Centro Universitário Ritter dos Reis, Porto Alegre, RS. E-mail: billminha@hotmail.com, Orcid: http://orcid.org/0000-0003-2704-2529

Marina Ferri Pezzini - Doutoranda e mestre em Ciências da Gastroenterologia/Hepatologia pela Universidade Federal do Rio Grande do Sul (UFRGS), Porto Alegre, RS. E-mail: mariinafp@ hotmail.com, Orcid: http://orcid.org/0000-0003-0475-6326

Julia Poeta- Mestre em Ciências Médicas pela Universidade Federal do Rio Grande do Sul e Docente no Centro Universitário Ritter dos Reis, Porto Alegre, RS. E-mail: julia_poeta@uniritter.edu.br, Orcid: http://orcid.org/0000-0002-2706-2207 
